\title{
Carbon Monoxide Releasing Molecule-3 Enhances Osteogenic Differentiation of Human Periodontal Ligament Stem Cells by Carbon Monoxide Release
}

\author{
Hui Chen ${ }^{1,2}$ \\ Yan Dai ${ }^{1,3}$ \\ Jing Cui ${ }^{4}$ \\ Xiaochun Yin ${ }^{2}$ \\ Wei Feng ${ }^{2}$ \\ Meiyi Iv ${ }^{1,5}$ \\ Hui Song' \\ 'Department of VIP Center, School and \\ Hospital of Stomatology, Cheeloo \\ College of Medicine, Shandong University \\ \& Shandong Key Laboratory of Oral \\ Tissue Regeneration \& Shandong \\ Engineering Laboratory for Dental \\ Materials and Oral Tissue Regeneration, \\ Jinan, 2500I2, Shandong, People's \\ Republic of China; ${ }^{2}$ Department of \\ Endodontics, Jinan Stomatological \\ Hospital, Jinan, Shandong Province, \\ People's Republic of China; ${ }^{3}$ Department \\ of Oral and Maxillofacial Surgery, Zibo \\ Central Hospital, Zibo, Shandong \\ Province, People's Republic of China; \\ ${ }^{4}$ Department of Oral and Maxillofacial \\ Surgery, Jinan Stomatological Hospital, \\ Jinan, Shandong Province, People's \\ Republic of China; ${ }^{5}$ Pediatric Dentistry, \\ Jinan Stomatological Hospital, Jinan, \\ Shandong Province, People's Republic of \\ China
}

Correspondence: Hui Song Department of VIP Center, School and Hospital of Stomatology, Cheeloo College of Medicine, Shandong University \&

Shandong Key Laboratory of Oral Tissue Regeneration \& Shandong Engineering Laboratory for Dental Materials and Oral Tissue Regeneration, No. 44-I Wenhua

Road West, Jinan, 2500I2, Shandong,

People's Republic of China

Tel +86 53I88382923

Fax +86 53188382923

Email songhui@sdu.edu.cn
Purpose: Limited intrinsic regeneration capacity following bone destruction remains a significant medical problem. Multiple regulatory effects of carbon monoxide releasing molecule-3 (CORM-3) have been reported. The aim of this study was to investigate the effect of CORM-3 on the osteogenic differentiation of human periodontal ligament stem cells (hPDLSCs) during osteogenesis.

Patients and Methods: hPDLSCs obtained from healthy periodontal ligament tissues were cultured and identified with specific surface antigens by flow cytometry. Effect of CORM-3 on the proliferation of hPDLSCs was determined by CCK-8 assay. Alizarin red staining and alkaline phosphatase (ALP) activity were used to assess the osteogenic differentiation of hPDLSCs. Real-time quantitative polymerase chain reaction (RT-qPCR) and Western blot analysis were used to detect the expression of the indicated genes. Critical-sized skull defect was made in Balb/c-nude mice, microcomputed tomography (Micro-CT) and Masson trichrome staining were used to assess the new bone regeneration in mice.

Results: CORM-3 (400 $\mu \mathrm{mol} / \mathrm{l})$ significantly promoted the proliferation of hPDLSCs. CORM-3 pretreatment not only notably enhanced the mRNA and protein expression of osteo-specific marker OPN, Runx2 and ALP, but also increased mineral deposition and ALP activity by the release of $\mathrm{CO}$ on day 3, 7 and $14(\mathrm{P}<0.05)$. Degassed CORM-3 did not show the same effect as CORM-3. In animal model, application of CORM-3 with hPDLSCs transplantation highly increased new bone formation in skull defect region.

Conclusion: CORM-3 promoted osteogenic differentiation of hPDLSCs, and increased hPDLSCs-induced new bone formation in mice with critical-sized skull defect, which suggests an efficient and promising strategy in the treatment of disease with bone defect.

Keywords: CORM-3, hPDLSC, osteo-specific marker, micro-computed tomography, osteopontin, Runx2

\section{Introduction}

Periodontitis (PD), a chronic periodontal disease characterized by irreversible and progressive degradation of periodontal supporting structures, alveolar bone defects and tooth loss. ${ }^{1}$ As the very important concerns not only in dental health but also in systemic health, periodontitis has been considered as the sixth most widespread disease worldwide. ${ }^{2}$ The ideal treatments for periodontitis not only include the inhibition of inflammation, but also the regeneration of periodontal tissues. Therapies based on stem cells increasingly become promising strategies in periodontal tissue regeneration. Periodontal ligament stem cells (PDLSCs) have self- 
renewal ability and multidifferentiation potency, ${ }^{3}$ which could be applied for the repair of periodontal bone defects in the context of the existing microenvironment in PD. ${ }^{4}$

Carbon monoxide (CO), a by-product of heme oxygenase catalyzing heme, has long been considered as a toxic gas. However, previous researches have shown that $\mathrm{CO}$ may have protective effects for the inhibition of cell apoptosis $^{5}$ and inflammation, ${ }^{6}$ induction of vasodilation ${ }^{7,8}$ and organ protection from ischemia/reperfusion injury. ${ }^{9,10}$ Newly synthesized transition metal carbonyl-compounds, $\mathrm{CO}$ release molecules (CORMs), are effective in regulating $\mathrm{CO}$ release in vivo and in vitro in appropriate circumstances. ${ }^{11}$ These $\mathrm{CO}$ releasing molecules can therefore be used as the new carrier to provide exogenous $\mathrm{CO}$. CORM-3 [tricarbonylchloro (glycinato) ruthenium (II)] is completely soluble in water with fast release of $\mathrm{CO}$ when dissolved in a physiological solution. ${ }^{12}$

By releasing CO, CORMs also induce an antiinflammatory effect. Our previous study showed that CORM-2 reduced periodontal inflammation and alveolar bone resorption in rats with experimental periodontitis. ${ }^{13} \mathrm{We}$ also reported CORM-3 suppressed the expression of adhesion molecules in human gingival fibroblasts co-stimulated by tumor necrosis factor- $\alpha$ and interleukin- $1 \beta,{ }^{14}$ and CORM-3 enhanced osteogenic differentiation of rat BMSCs. ${ }^{15}$

To date, the effects of CORM-3 on osteogenesis of hPDLSCs have rarely been reported. In this study, we used human PDLSCS as an in vitro model to investigate the effect of CORM-3 on the osteogenic differentiation of hPDLSCs, we also applied the balb/c-nude mice with critical-sized skull defect as in vivo model to explore the effect of CORM-3 on the hPDLSCs-induced bone regeneration.

\section{Materials and Methods}

\section{Isolation and Culture of hPDLSCs}

All experiments were approved by the Ethics Committee of the School and Hospital of Stomatology, Shandong University (Jinan, China) (NO.202000104). The patients of 11-18-yearold without systemic disease were selected for this study. Prior to the surgery, written informed consents were signed by the patients and their guardians in writing. 10 premolars and 8 impacted wisdom teeth removed for orthodontic treatment were collected for cell isolation. The methods and procedure of hPDLSCs isolation and culture were processed as previously reported. ${ }^{16,17}$ Briefly, the extracted teeth were stored in a sterile centrifuge tube containing $\alpha$-MEM (HyClone, GE
Healthcare Life Sciences, Logan, UT, USA) supplemented with streptomycin $100 \mathrm{mg} / \mathrm{mL}$ and penicillin $\mathrm{G} 100 \mathrm{U} / \mathrm{mL}$ (Sigma-Aldrich, St Louis, MO, USA) on ice, and then were immediately transported from the hospital to the laboratory. The periodontal ligament tissues in the middle third of the tooth root were scraped off, and were then cut into tiny pieces and put into the $25 \mathrm{~cm} 2$ culture bottles (Corning Inc., Corning, NY, USA) containing $4 \mathrm{~mL} \alpha$ MEM supplemented with 100 $\mathrm{U} / \mathrm{mL}$ penicillin, $100 \mathrm{mg} / \mathrm{mL}$ streptomycin and $20 \%$ fetal bovine serum (Gibco; Thermo Fisher Scientific, Inc.), in an incubator at $37^{\circ} \mathrm{C}$ with $5 \% \mathrm{CO}_{2}$. hPDLSCs for the study were obtained by colony formation assay. Cells of passages $2-5$ were used in the study. Isolation and culture of hPDLSCs was conducted in accordance with the Declaration of Helsinki.

\section{Identification of Isolated hPDLSCs by Flow Cytometry}

After reaching $90 \%$ confluence, hPDLSCs were trypsinized with $0.25 \%$ Trypsin containing $0.02 \%$ EDTA (HyClone; GE Healthcare Life Sciences), and rinsed by phosphate-buffered saline (PBS; Thermo Fisher Scientific, Inc.) for three times. The cells were then resuspended in $0.5 \mathrm{~mL}$ PBS, and incubated at dark with fluorescent dye-conjugated monoclonal antibody on ice for $20 \mathrm{~min}$. Cells were analyzed using a FACSCalibur flow cytometer (BD Biosciences, Franklin Lakes, NJ, USA) with a BD Stemflow ${ }^{\mathrm{TM}}$ hMSC Analysis kit for the detection of human CD90, CD44, CD105, CD34 and CD45 (BD Falcon) expression.

\section{Cell Proliferation by Cell Counting Kit-8 (CCK-8) Assay}

The cell proliferation and the toxicity of different concentration of CORM-3 on the hPDLSCs was detected by Cell Counting Kit8 (CCK-8; Dojindo Molecular Technologies, Inc., Beijing, China). P3 hPDLSCs were inoculated in 96well plates (5000 cells/well) and cultivated in control medium for 1 day at $37^{\circ} \mathrm{C}$. The medium was refreshed with CORM-3 medium at the concentration of 0 (control group), 100, 200, 400 and $800 \mu \mathrm{M}$, respectively. Following $24 \mathrm{~h}$ incubation, $10 \mu \mathrm{L}$ CCK8 reagent was added to each well. Following $2 \mathrm{~h}$ incubation, the cells were measured for spectrophotometric absorbance at OD450 nm (optical density) by using a microplate reader (Spectro Analytical Instruments GmbH, Kleve, Germany). The assay was done in triplicate and each experiment was repeated three times. 


\section{Multiple Differentiation Potency of hPDLSCs}

The P3 hPDLSCs were seeded in 6-well plates $\left(2 \times 10^{5}\right.$ cells/ well). When reaching $90 \%$ confluence, the medium was refreshed with osteogenic induction medium or adipogenic induction medium for cell differentiation. For osteogenic differentiation, hPDLSCs were incubated with osteogenic induction medium (OIM) (Cyagen Biosciences Inc., Guangzhou, China) containing $0.2 \mathrm{mM}$ ascorbic acid, $10 \mathrm{mM} \beta$ glycerophosphate, $10^{-7} \mathrm{M}$ dexamethasone; $10 \%$ FBS, and $100 \mathrm{U} / \mathrm{mL}$ penicillin-streptomycin at $37^{\circ} \mathrm{C}$. Cells grown in the medium supplemented with $10 \%$ FBS and $100 \mathrm{U} / \mathrm{mL}$ penicillin-streptomycin (control medium) were used as control. After 3 weeks of induction, cells were fixed with $4 \%$ paraformaldehyde for $30 \mathrm{~min}$ at $37^{\circ} \mathrm{C}$. The cells were washed with PBS for three times, and then incubated with $2 \%$ Alizarin red S (pH 4.3) (AR; Sigma-Aldrich; Merck KGaA) at room temperature for $5 \mathrm{~min}$, followed by another three washes. The formation of mineralized nodules was observed using an inverted microscope (Olympus Corp., Tokyo, Japan). The relative amount of calcium was quantified using $10 \mathrm{mM}$ sodium phosphate solution and $10 \%(\mathrm{w} / \mathrm{v})$ cetylpyridinium chloride (CPC) (Solarbio). The absorbance at wavelength of $562 \mathrm{~nm}$ was measured.

For adipogenic differentiation, P3 cells were cultured in the adipogenic induction medium containing $100 \mathrm{U} / \mathrm{mL}$ penicillin-streptomycin, $10 \% \mathrm{FBS}, 2 \mu \mathrm{mol} / 1$ dexamethasone (Beijing Solarbio Science \& Technology Co., Ltd.), $0.2 \mathrm{mmol} / 1$ indomethacin, $0.01 \mathrm{~g} / \mathrm{l}$ insulin and $0.5 \mathrm{mmol} / 1$ isobutylmethylxanthine (all Sigma-Aldrich; Merck KGaA) at $37^{\circ} \mathrm{C}$. Cells grown in the medium supplemented with $10 \% \mathrm{FBS}$ and $100 \mathrm{U} / \mathrm{mL}$ penicillin-streptomycin (control medium) were used as control. Following 3 weeks of culture, cells were fixed with $4 \%$ paraformaldehyde and washed with PBS, cells were then incubated with Oil Red O (Cyagen Biosciences, Suzhou, China) at room temperature for $30 \mathrm{~min}$ and subsequently washed with PBS three times. The lipid droplets in the cells were detected using an inverted microscope (Olympus Corp.)

\section{Effects of CORM-3 on Osteogenic Differentiation of hPDLSCs}

The hPDLSCs were divided into four groups. Osteogenic group, in which the cells were cultured in osteogenic induction medium at room temperature; CORM-3+osteogenic group, in which the cells were pretreated with 400 $\mu \mathrm{M}$ CORM-3 at room temperature for $24 \mathrm{~h}$, and subsequently the medium was completely refreshed with osteogenic induction medium; Degassed CORM-3+osteogenic group, in which the cells were pretreated with 400 $\mu \mathrm{M}$ degassed CORM-3 for $24 \mathrm{~h}$, and then replaced with osteogenic medium; Control group, in which the cells were cultivated in the control medium. The medium of all groups was refreshed every three days. CORM-3 medium was prepared freshly before the experiment. Degassed CORM-3 was prepared by placing CORM-3 medium in a $50 \mathrm{~mL}$ sterile centrifuge tube in an incubator at room temperature with the lid open for $24 \mathrm{hrs}$ prior to the experiment. As the negative control, degassed CORM-3 group was designed to evaluate whether $\mathrm{CO}$ was involved in the pharmacological activity of CORM-3. The mRNA and protein expression of osteogenesis-related factor Runx2, ALP, OPN (encoding gene SPP1, NM_000582.3), and ALP activity and mineralization deposits were detected on the 3rd, 7th and 14th day.

\section{RT-qPCR Analysis}

On the 3rd, 7th and 14th day, $1 \mathrm{~mL}$ TRIzol (Takara, Kusatsu, Japan) was added to each well of all groups and total RNA was extracted according to the manufacturer's protocol (Invitrogen; Thermo Fisher Scientific, Inc). RNA concentration was assessed by an ultramicro spectrophotometer (Thermo Fisher Scientific, Waltham, USA). The cDNA was synthesized from total RNA (1 $\mu \mathrm{g})$ using a PrimeScript Reverse Transcriptase reagent kit (Takara Biotechnology Co., Ltd., Dalian, China), according to protocol. The cDNA samples were then amplified in $20 \mu \mathrm{L}$ reaction system containing SYBR Green (Takara Biotechnology Co., Ltd.) on a Roche Light Cycler $^{\circledR} 480$ (Hoffmann-La Roche Ltd., Basel, Switzerland) for the gene expression of ALP, Runx2 and SPP1 (encoding gene of OPN, NM_001251830.2). The RTqPCR thermal cycling was as follows: initial denaturation at $95^{\circ} \mathrm{C}$ for $30 \mathrm{sec}, 40 \mathrm{cycles}$ of $95^{\circ} \mathrm{C}$ for $5 \mathrm{sec}$ and then $60^{\circ} \mathrm{C}$ for $20 \mathrm{sec}$. The $2-\Delta \Delta \mathrm{Ct}$ method was used to analyze the relative gene expression results, ${ }^{18}$ and the gene expression was normalized with GADPH level. The sequences of specific primers used were as follows: GAPDH, forward 5'-GCA CCG TCA AGG CTG AGA AC-3' and reverse 5'-TGG TGA AGA CGC CAG TGG A-3'; Runx2, forward 5'-TCCACACCAT TAGGGACCATC-3' and reverse 5'-TGCTAATGCTTC GTGTTTCCA-3'; ALP, forward 5'-ATGGGATGGGTGTCT CCACA-3' and reverse5'-CCACGAAGGGGAACTTGTC -3'; SPP1, forward 5'-TCCTAGCCCCACAGACCCTT-3' and reverse 5'-CACACTATCACCTCGGCCAT-3' 


\section{Western Blot Analysis}

Following 3, 7 and 14 days of cultivation, cells were rinsed with ice-cold PBS and lysed with radio immunoprecipitation assay lysis buffer (Beyotime Institute of Biotechnology, Jiangsu, China) containing $1 \%$ phenylmethanesulfonyl fluoride protease inhibitors $(1 \mathrm{mmol} / \mathrm{l})$ (Solarbio). Total protein concentration of each group was quantified with a Bicinchoninic Acid Protein Assay kit (Beyotime Institute of Biotechnology, Jiangsu, China). Subsequently, Protein samples $(20 \mu \mathrm{g} / \mathrm{lane})$ were separated electrophoretically with $12 \%$ SDS-PAGE (Boster, Wuhan, China) and then electroblotted onto PVDF membranes (Bio-Rad Laboratories, Hercules, USA). Following blocked with 5\% nonfat milk for $1 \mathrm{~h}$ at $37^{\circ} \mathrm{C}$, the membranes were incubated at $4{ }^{\circ} \mathrm{C}$ overnight with the primary antibodies: mouse anti-GAPDH monoclonal antibody (cat no., HRP-60004, Proteintech) (1:10,000 dilution); rabbit anti-Runx2 Polyclonal antibody (cat no., ab23981, Abcam) (1:1000 dilution); rabbit anti-ALP monoclonal antibody (cat no., ab108337, Abcam) (1:10,000 dilution); and rabbit anti-OPN Polyclonal antibody (cat no., ab8448, Abcam) (1:1000 dilution), respectively. Membranes were washed with TBST for three times, and then incubated with horseradish peroxidase-labeled secondary antibodies (cat. nos., SA00001-2; Proteintech Group, Inc., Rosemont, IL, USA) (1:10,000 dilution) at room temperature for $1 \mathrm{~h}$. The immunoreactive protein bands were visualized using the chemiluminescent HRP substrate (EMD Millipore, Billerica, USA) and the densities of the band were quantified using ImageJ 1.45V software (NIH, Bethesda, MD, USA).

\section{Alkaline Phosphatase (ALP) Activity Assay} Cells were rinsed twice with pre-cold PBS and scraped off, then lysed with $1 \%$ Triton (Solarbio) for half an hour. Subsequently, the collected cell lysates were centrifuged at $4^{\circ}$ $\mathrm{C}$ for $15 \mathrm{~min}$ at $12,000 \mathrm{xg}$. The protein concentration of the collected cell lysates was measured with a Bicinchoninic Acid Protein Assay kit (Solarbio). The ALP activity was detected according to the instructions of the ALP activity assay kit (Nanjing Jiancheng Bioengineering Institute, Nanjing, China). OD values were measured at a wavelength of 520 $\mathrm{nm}$ using an ELISA plate reader. ALP activity was calculated and expressed as King-Armstrong units/g of total cellular protein.

\section{Analysis of Mineralization of hPDLSCs}

Alizarin red S (pH 4.3, Sigma Aldrich) was used to evaluate the extracellular matrix calcification As mentioned above, staining of the cells in the well was dissolved in $100 \mu \mathrm{M}$ cetylpyridinium chloride (CPC) at room temperature for $1 \mathrm{~h}$. For the evaluation, the absorbance was detected at a wavelength of $562 \mathrm{~nm}$ on ELISA plate reader.

\section{Experimental Animals}

Twenty-four seven-week-old male balb/c-nude mice (weight 20-25g, Beijing Vital River Laboratory Animal Technology Co., Ltd.) were domesticated for 7 days prior to the experiment and maintained on water ad libitum and a normal hard food diet. Nude mice were randomly divided into 4 groups. CORM-3+hPDLSC group and normal saline+hPDLSC group: defects were filled with collagen membrane seeded with hPDLSCs, in the presence (CORM-3+hPDLSC group) or absence (normal saline+ hPDLSC group) of CORM-3, respectively; CORM-3 group: defects were filled with collagen membrane with the application of CORM-3; and normal saline group (control group): defects were filled with collagen membrane without application of CORM-3. The experiment on nude mouse was performed in accordance with the $3 \mathrm{R}$ principle of the guidelines for the Care and Use of Laboratory Animals and authorized by Animal Care and Use Medical Ethical Committee of School and Hospital of Stomatology, Shandong University. (NO. 20190802)

\section{Cell Seeding Before Skull Defect Surgery}

Collagen membranes (Zhenghai Bio-tech, Co., Ltd, Yantai, China) were cut into $4 \mathrm{~mm}$ diameter round pieces and sterilized for use. The hPDLSCs $\left(1 \times 10^{5}\right.$ P2) were suspended in $10 \mu \mathrm{L} \alpha$-MEM medium containing $10 \%$ fetal bovine serum (FBS), and inoculated on the surface of the collagen membrane, followed by $12 \mathrm{~h}$ culture in the incubator at $5 \% \mathrm{CO}_{2}$ at $37^{\circ} \mathrm{C}$.

\section{Skull Defects Operation}

Twenty-four balb/c-nude mice (20-25g) were anesthetized by intraperitoneal injection with $10 \%$ chloral hydrate (400 $\mathrm{mg} / \mathrm{lkg}$ body weight). No toe contraction reflex demonstrated that the nude mice were completely anesthetized. A vertical cut in the middle of the scalp was made under sterile condition. The skull was exposed, an outer diameter of $4 \mathrm{~mm}$ full-thickness skull defect on the bilateral areas was created with a bone trephine (Nobel, Sweden). Collagen membranes with or without hPDLSCs were transferred into the defect areas of the animals in different groups. Elevated flaps were restored and sutured with absorbable 
suture line. After surgery, the heartbeat and respiration of the animals were monitored until the animals woke up.

After operation, penicillin sodium (500,000 units $/ \mathrm{kg})$ was injected intramuscularly for 3 days, the wound healing and mental status of the animals was observed at any time. Abnormal behavior or post-operative complications was monitored. Animals had free access to water and food. No mouse exhibited signs of peritonitis after the administration of $10 \%$ chloral hydrate.

Intraperitoneal administration of CORM-3 was started on the 3rd day after surgery and performed every other day. CORM-3 dissolved in $1 \mathrm{~mL} 0.9 \%$ normal saline (10 $\mathrm{mg} / \mathrm{kg}$ ) was injected intraperitoneally in CORM-3 + hPDLSC group and CORM-3 group, while $0.9 \%$ normal saline $(1 \mathrm{~mL})$ was in the hPDLSC+normal saline group and normal saline group. For nude mice in CORM-3+hPDLSC group and CORM-3 group, the body weights of the animals were recorded before each administration.

During the experiment, when the following humane endpoints were reached, the nude mice were euthanized by means of cervical dislocation: i) mice for weight loss (15-20\% rapidly decrease of the original body weight); ii) weakness (incapable of eating and drinking on their own); iii) anorexia (not any food at all within 24-36 hours); iv) body organ infection (treatment of penicillin was ineffective). The mice were confirmed to be dead based on cardio-respiratory arrest for 2 minutes, and no blink reflex.

\section{Tissue Preparation}

Four weeks and seven weeks after operation, the mice were euthanized. The skull was dissected and placed in $4 \%$ paraformaldehyde and fixed at $4{ }^{\circ} \mathrm{C}$ for $24-48$ hours.

\section{Masson Trichrome Staining}

Trichromatic staining of Masson was carried out using Masson staining Kit (Jiancheng Biological Inc, Nanjing, China). ${ }^{19}$ Tissues from defect regions were examined and photographed by an optical microscope at the same magnification. Imagepro-plus6.0 (Media Cybernetics, Inc. MD, USA) was used to analyze newly formed bone regions.

\section{Hematoxylin and Eosin Staining and Micro-CT Detection}

Hematoxylin and eosin staining were performed according to the standard protocols. Briefly, the sections were dewaxed by xylene, hydrated in gradient ethanol, and then treated with hematoxylin to stain the nuclei for 15 minutes. Subsequently, the sections were added with eosin staining solution. The slices were sealed with a transparent mountant and a coverslip. The morphology of the tissue was observed under the microscope. Image-Pro-Plus 6.0 software was used for the measurement and analysis of areas of new bone formation. The skulls were detected in three dimensions using Micro-CT ( $\mu \mathrm{CT}-100$; SCANCO Medical AG, Switzerland) and scanned at $65 \mathrm{kV} /$ $380 \mu \mathrm{A}$ as mentioned previously. ${ }^{20}$ For animals of each group, three fixed specimens were randomly selected for micro-CT scans. A $4 \mathrm{~mm}$ diameter circular region was selected as the region of interest (ROI) in the skull defect area. The skull defect area was three-dimensionally reconstructed using the V6.5-3 analysis software. Volume measurement was performed after selecting a 3D area of interest (ROI) based on skull defect. The microstructural parameters such as the bone volume fraction $(\mathrm{BV} / \mathrm{TV} \%)$ of the new bone, the number of trabecular bone (Tb.N, mm-1), the trabecular separation (Tb. $\mathrm{Sp}, \mathrm{mm}$ ) and the thickness of trabecular (Tb.Th, $\mathrm{mm}$ ) were simultaneously analyzed using software (CT Analyser; Bruker). The measurement was repeated for 3 times.

\section{Statistical Analysis}

All data were presented as mean \pm standard deviation (SD). The significance of difference between two groups was statistically analyzed by one-way ANOVA followed by the Turkey's post hoc test. $\mathrm{P}<0.05$ was set as a significant difference.

\section{Results}

\section{Identification of Human PDLSCs}

hPDLSCs were identified by cell morphology and specific cell surface antigen. As shown in Figure 1A, the primary cultured hPDLSCs exhibited a typical spindle-shaped fibroblast-like morphology. The osteogenic differentiation or adipogenic differentiation was testified by the formation of mineralized nodules (Figure 1B and C) or lipid droplets (Figure 1F and G) following 21 days of induction. A cluster of cells obtained from a single hPDLSC is shown in Figure 1E. Flow cytometric analysis showed the cultured hPDLSCs lowly or negatively expressed CD45 (0.16\%) and CD34 (1.39\%), whereas highly and positively expressed CD44 (99.9\%), CD90 (99.9\%) and CD105 (99.7\%) (Figure 1D).

\section{Effect of CORM-3 on hPDLSC Proliferation}

The hPDLSCs were treated with CORM-3 at different concentrations. The effect of CORM-3 on hPDLSC proliferation was assessed using a CCK-8 proliferation assay. As indicated in Figure 2, 100, 200 and $400 \mu \mathrm{M}$ CORM-3 treatment 
promoted the proliferation of hPDLSCs, however, only 400 $\mu \mathrm{M}$ CORM-3 showed the significant promotive effect $(\mathrm{P}<0.05) .800 \mu \mathrm{M}$ CORM-3 treatment induced a clear decrease in cell viability, compared with the control group $(\mathrm{P}<0.05)$. Based on these data, $400 \mu \mathrm{M}$ CORM-3 was selected as the working concentration for the following experiments.

\section{ALP Activity of hPDLSCs During Osteogenic Differentiation}

After treated with $400 \mu \mathrm{M}$ CORM-3 for 3, 7 and 14 days, the ALP activity of hPDLSCs was assessed
(Figure 3). ALP activity in the CORM-3+osteogenic group showed significantly higher than that in other groups on day 3,7 and $14(\mathrm{P}<0.001)$. ALP activity was also increased in the osteogenic group and degassed CORM-3+osteogenic group, compared with the control group $(\mathrm{P}<0.05)$. Notably, there were no significant differences in ALP activity between the degassed CORM-3+osteogenic group and the osteogenic group at each time-point, suggesting that the effect of CORM-3 on ALP activity depended on the released CO (Figure 3).
A

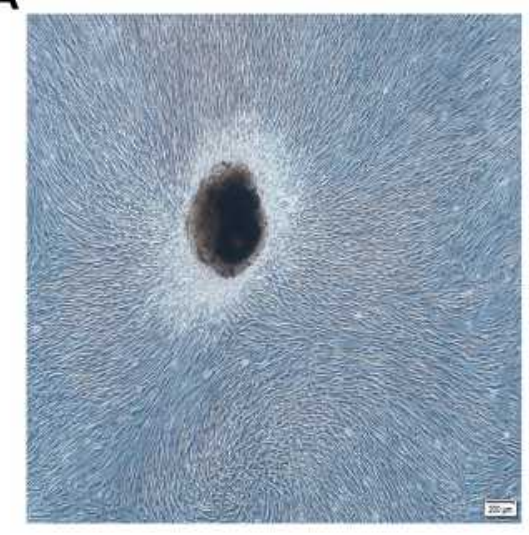

B

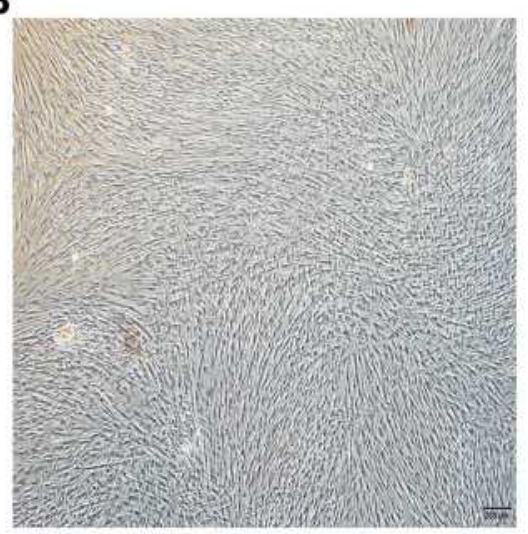

C

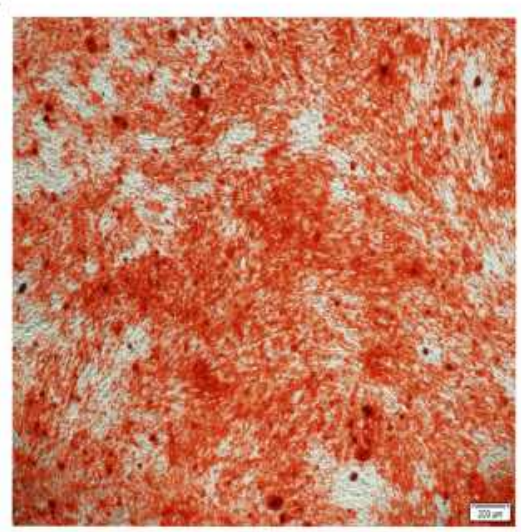

D
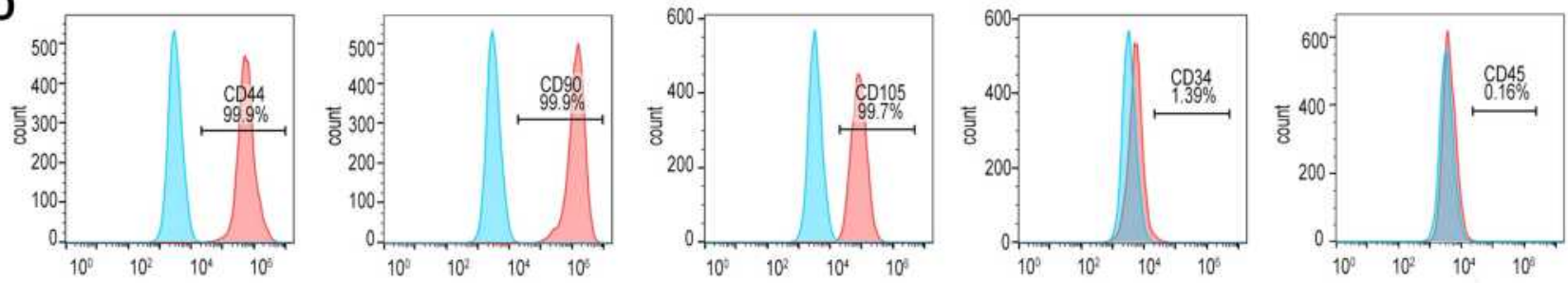

E

$\mathbf{F}$
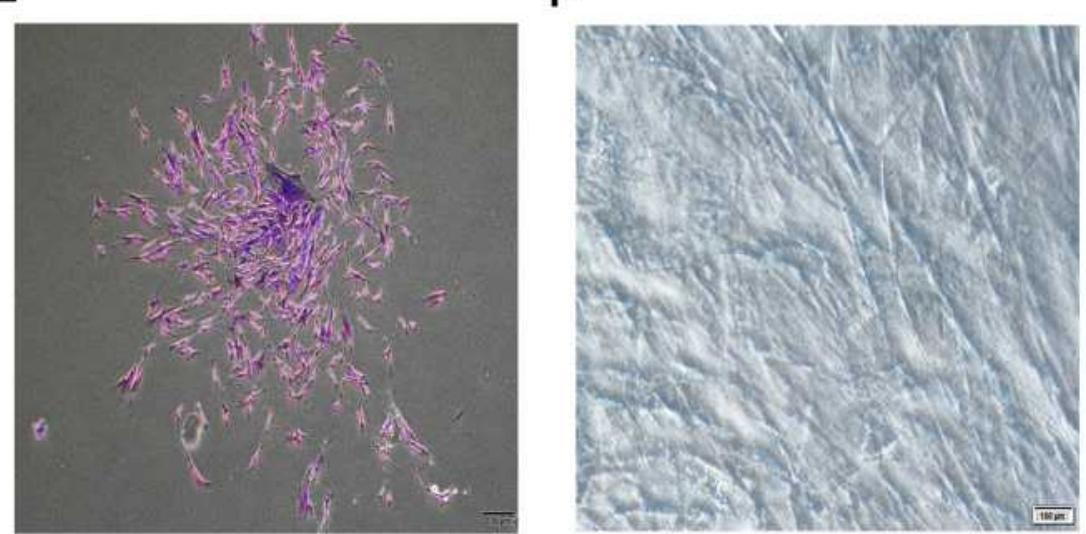

G

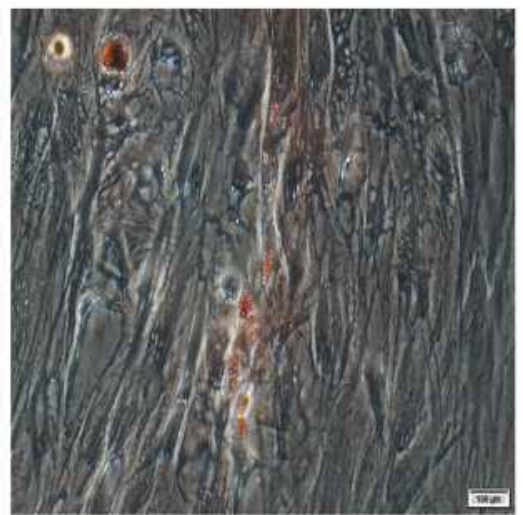

Figure I Characterization of hPDLSCs. (A) hPDLSCs grown from the periodontal ligament tissue mass were cultured in the normal medium, cell morphology was assessed by a microscope. (B) and (C) hPDLSCs were cultured in control medium (B) or osteogenic induction medium (C) for 21 days. Then the cell mineralization was checked by Alizarin red S staining. (D) The surface expression of CD44, CD90, CDI05, CD34 and CD45 was assessed by flow cytometry. (E) hPDLSCs were isolated by using limiting dilution to form single cloning. (F and $\mathbf{G}$ ) hPDLSCs were induced in control medium (F) or adipogenic induction medium (G) for 21 days. Then the cell adipogenic differentiation was examined by Oil red $O$ staining. 


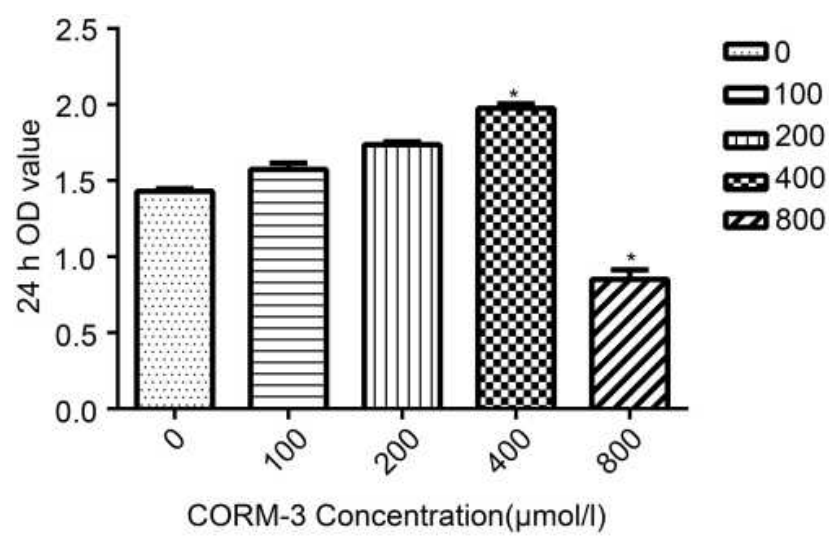

Figure 2 Effect of CORM-3 on the proliferation of hPDLSCs. hPDLSCs were incubated in the medium in the presence of CORM-3 at the concentration of $100,200,400$ and $800 \mu \mathrm{M}$, respectively. Cells grown in the normal medium were used as control. After $24 \mathrm{~h}$, the cell proliferation was assessed by CCK-8 kit. The experiment was repeated for at least three times. Data were expressed as the mean OD value \pm standard deviation $(n=3)$. $* P<0.05$ vs $0 \mu \mathrm{mol} / /$ group.

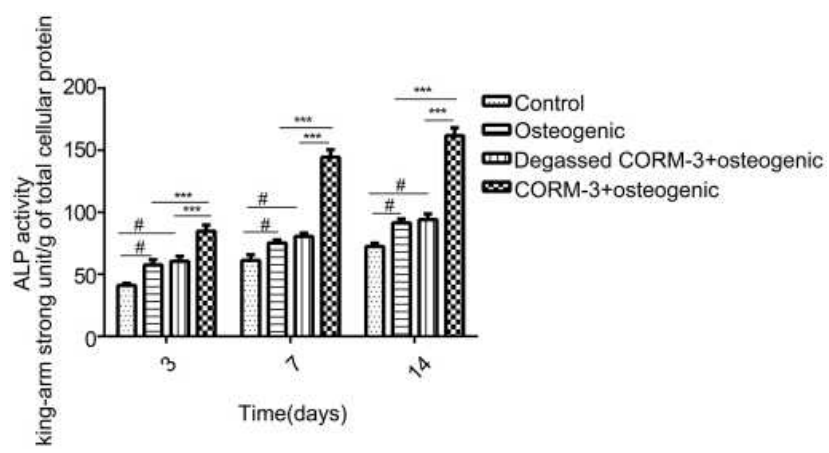

Figure 3 Effect of CORM-3 on ALP activity. hPDLSCs were divided into four groups. Control group, in which the cells were cultivated in the control medium; osteogenic group, in which the cells were cultured in osteogenic induction medium; degassed CORM-3+osteogenic group, in which the cells were pretreated with $400 \mu \mathrm{M}$ degassed CORM-3 for $24 \mathrm{~h}$, then replaced with osteogenic medium; CORM-3 +osteogenic group, in which the cells were pretreated with $400 \mu \mathrm{M}$ CORM-3 for $24 \mathrm{~h}$, and subsequently the medium was changed with osteogenic induction medium. On day 3, 7, and I4, the ALP activity of the cells was assessed. Data were expressed as the mean \pm standard deviation $(\mathrm{n}=3) .{ }^{\#} \mathrm{P}<0.05$ vs Control; $* * * \mathrm{P}<0.00 \mathrm{I}$ as indicated.

\section{Effect of CORM-3 on mRNA and Protein Expression of ALP, Runx2, and OPN During Osteogenic Differentiation}

In order to investigate the effect of CORM-3 on osteogenic differentiation in hPDLSCs, the mRNA expression of SSP1, Runx2 and ALP were detected by RT-qPCR on day 3, 7 and 14 during osteogenic differentiation. As shown in Figure 4, in the CORM-3+osteogenic group, the mRNA expression of the three osteogenic markers increased significantly at all time points examined, compared with that in other groups. The highest mRNA expression of SSP1, Runx2 and ALP occurred on day 14. In consistent with the mRNA expression results, the cells in the CORM-3+osteogenic group expressed significantly
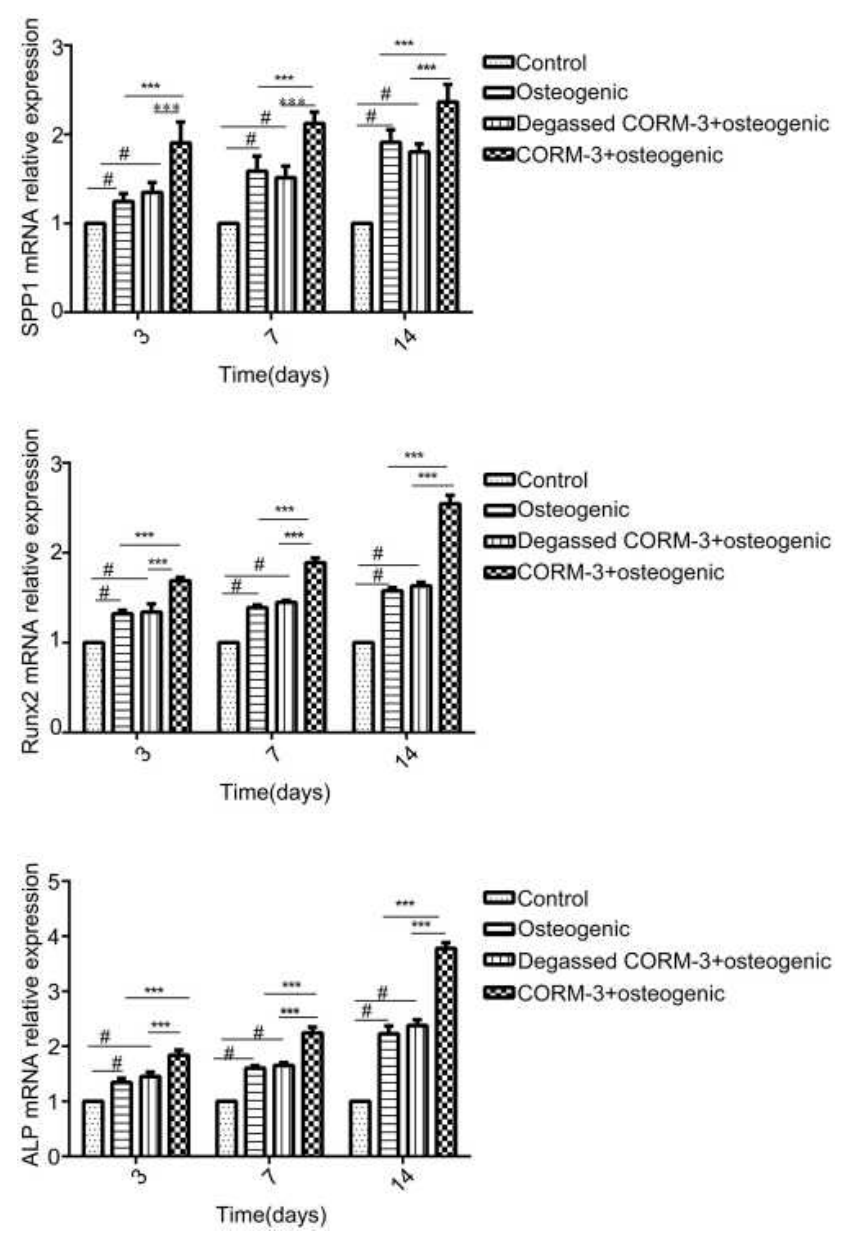

Figure 4 Effect of CORM-3 on the mRNA expression of osteogenesis-specific genes. hPDLSCs were divided into four groups. Control group, osteogenic group, degassed CORM-3+osteogenic group, and CORM-3+osteogenic group, as described above. The mRNA expression of SSPI, Runx2 and ALP was determined by RT-qPCR on day 3, 7 and 14. Data were presented as the mean \pm standard deviation $(\mathrm{n}=3)$. ${ }^{\#} \mathrm{P}<0.05$ vs Control; $* * * \mathrm{P}<0.001$ as indicated.

higher protein level of OPN, ALP and Runx2 than other group on day 3, 7 and $14(\mathrm{P}<0.001)$. In the CORM-3+osteogenic group, the expression of Runx 2 was maximally increased by 3.5-fold compared to the control group, and 2-fold with the osteogenic group on day $14(\mathrm{P}<0.001)$ (Figure $5 \mathrm{~A}$ and $\mathrm{B}$ ).

The cells in the degassed CORM-3+osteogenic group showed no significantly different mRNA and protein expression of Runx2, ALP and OPN, compared to the osteogenic group, suggesting that the regulatory effect of CORM-3 was mediated by the released CO.

\section{CORM-3 Increased Mineralization of hPDLSCs}

HPDLSCs in different groups were cultured in the conditions as indicated for 14 and 21 days, respectively. Extracellular matrix calcification of the cells was examined by AR staining 

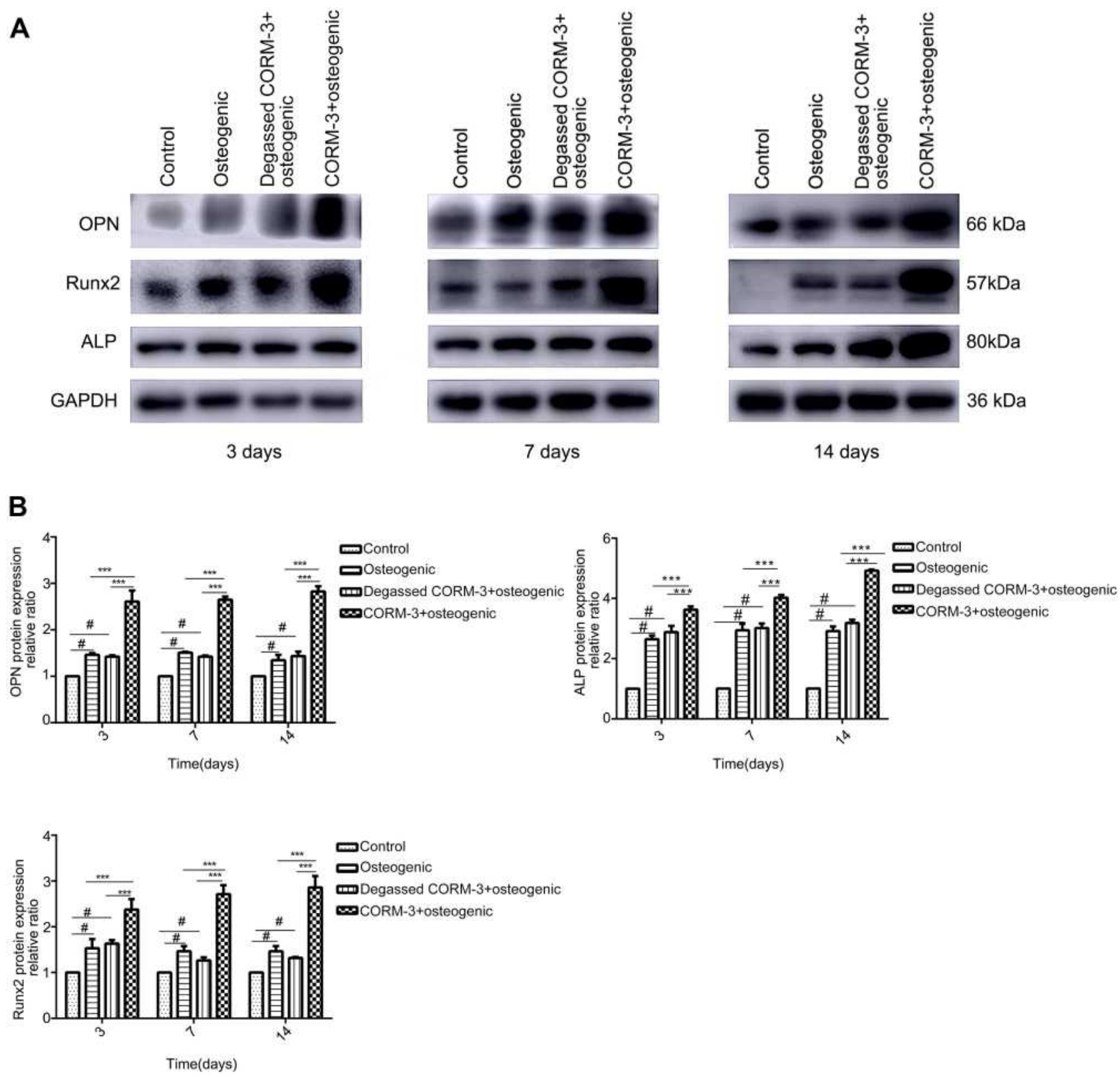

Figure 5 Effect of CORM-3 on the protein expression of osteogenesis-specific factors. hPDLSCs were divided into four groups. Control group, osteogenic group, degassed CORM-3+osteogenic group, and CORM-3+osteogenic group, as described above. The protein expression of OPN, Runx2 and ALP was determined by Western blot on day 3, 7 and 14. (A) Representative Western blot images of the OPN, Runx2 and ALP protein expression of the three independent experiments. (B) Quantitative results of Western blot images using ImageJ software. Data were presented as the mean \pm standard deviation $(\mathrm{n}=3)$. ${ }^{*} \mathrm{P}<0.05$ vs Control; *** $\mathrm{P}<0.00 \mathrm{I}$ as indicated.

(Figure 6A). As shown in Figure 6B, the mineralization of the cells in the CORM-3+osteogenic group was significantly higher than that in other three groups on the 14th and 21 st day $(\mathrm{P}<0.001)$. The calcium deposition in the osteogenic group increased significantly, compared with that in the control group $(\mathrm{P}<0.05)$. The mineralization in the degassed CORM-3 +osteogenic group showed no significant difference with osteogenic group, indicating that the effect of CORM-3 on osteogenic differentiation was through $\mathrm{CO}$ release (Figure 6B).

\section{CORM-3 with hPDLSC Transplantation Enhanced New Bone Formation in Nude Mice with Critical-Sized Skull Defect}

Skull defect with an outer diameter of $4 \mathrm{~mm}$ was established and collagen membrane (with or without hPDLSCs plantation) was transplanted into the defect region. HE staining and Masson staining were used to evaluate the new bone mass in the skull defect region (Figure 7A). HE staining showed that 4 weeks after surgery, a large piece of new bone was found in the 

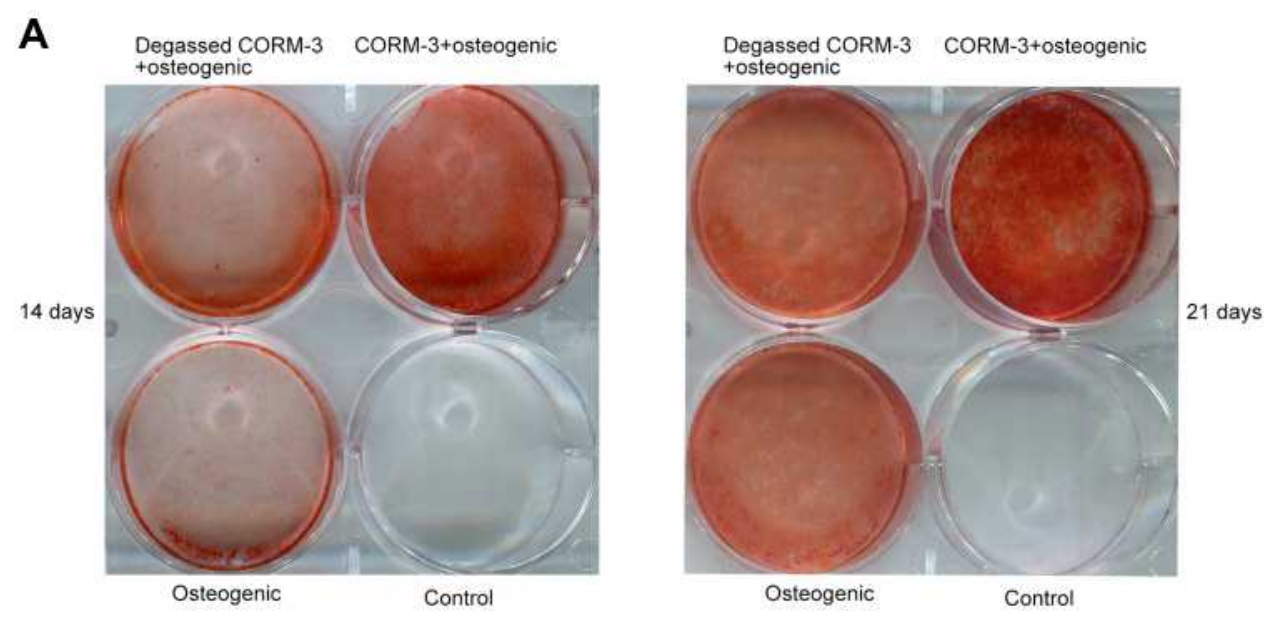

B

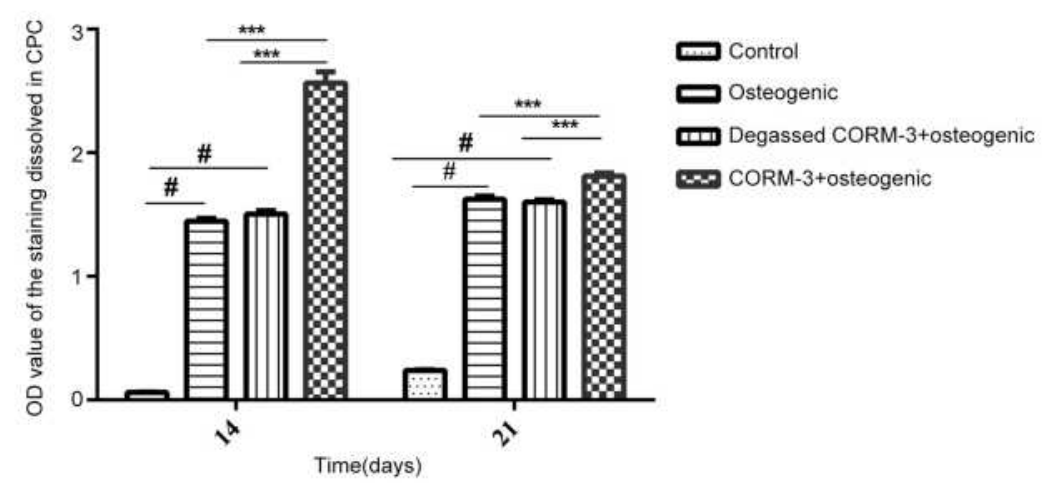

Figure 6 Effect of CORM-3 on cell mineralization during osteogenic differentiation of hPDLSCs. hPDLSCs were divided into four groups. Control group, osteogenic group, degassed CORM-3+osteogenic group, and CORM-3+osteogenic group, as described above. (A) On day 7 and 14 , the mineralization of the cells was checked by alizarin red staining. (B) Quantitative results of alizarin red staining in all groups. Data were presented as the mean \pm standard deviation $(n=3)$. ${ }^{\#} \mathrm{P}<0.05$ vs Control; ${ }^{* * *} \mathrm{P}<0.00 \mathrm{I}$ as indicated.

defect region in CORM-3+hPDLSC group, the area ratio of new bone measured by Image-Pro-Plus $6.0 \mathrm{~m}$ software was 1.7 , 1.54, and 16 times higher than that in CORM-3 group, normal saline + hPDLSC group and control group, respectively. The difference was statistically significant $(\mathrm{P}<0.05)$ (Figure $7 \mathrm{~B})$. The percentage of newly formed bone in CORM-3 group and normal saline $+\mathrm{hPDLSC}$ group was significantly higher than that in control group $(\mathrm{P}<0.05)$. No obvious new bone was found in the control group, only some cells were scattered in the bone defect area.

As shown in Figure 7C, Masson staining results showed the mineralization of newly formed bone in different groups. The red dye area represented the mineralized bone and the blue dye area represented mineralizing bone. The new bone mass in CORM-3+hPDLSC group was notably higher than that in other three groups $(\mathrm{P}<0.05)$ (Figure 7D). New bone mass in CORM-3 group and normal saline+hPDLSC group was much higher than that in normal saline group (control group) $(\mathrm{P}<0.05)$.
CORM-3 with hPDLSC Transplantation Increased the Bone Defect Repair in Nude Mice with Critical-Sized Skull Defect

As shown in Figure 8A, collagen membranes (engrafted with or without hPDLSCs) were transplanted into the defect area. Micro-CT analysis showed that larger high-density areas were found in skull defect region of animals in CORM-3+hPDLSC group (Figure 8B). Further measurement and quantitative analysis of the region of interest (ROI) revealed that the bone volume fraction (BV/TV) in CORM-3+hPDLSC group was significantly higher than that in other three groups, which was $1.41,1.33$, and 2 times higher than that in CORM-3 group, normal saline + hPDLSC group and control group, respectively. Both number of trabeculae (Tb.N) and Tb.Th (Figure 8C) in CORM-3+hPDLSC group were the highest in four groups $(\mathrm{P}<0.05)$, whereas Tb.Sp (Figure $8 \mathrm{C}$ ) was significantly lower than that in other three groups $(\mathrm{P}<0.05)$. There was no significant difference of Tb.Sp between CORM-3 group and normal saline+hPDLSC group. 
A

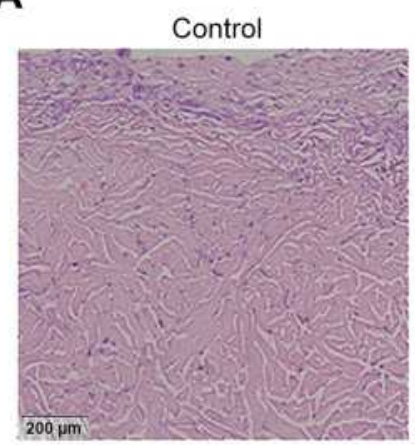

CORM-3

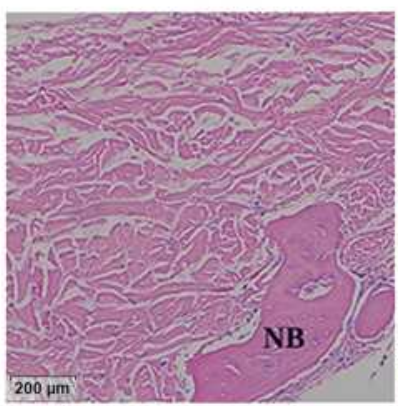

C

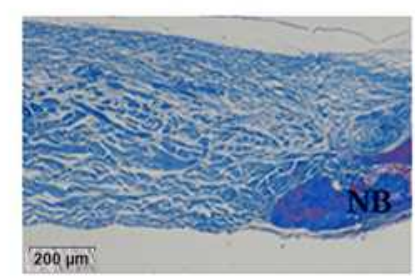

CORM-3

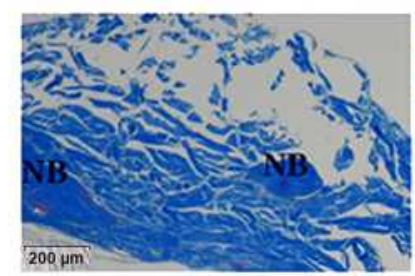

Normal saline+hPDLSC

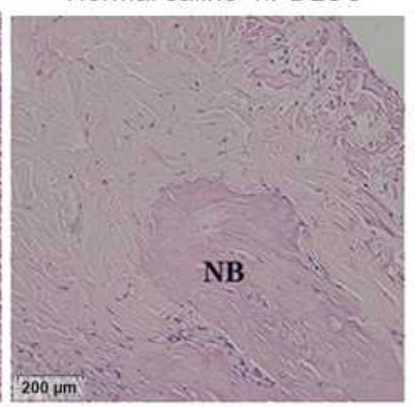

CORM-3+hPDLSC

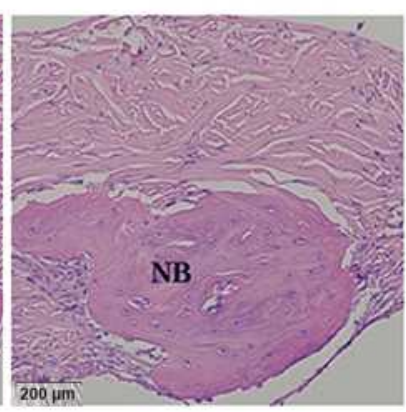

Normal saline+hPDLSC

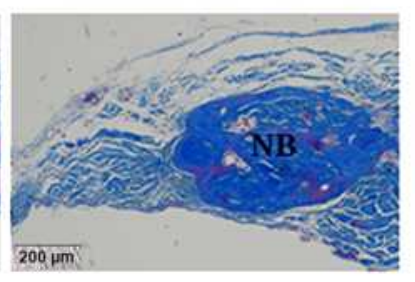

CORM-3+hPDLSC

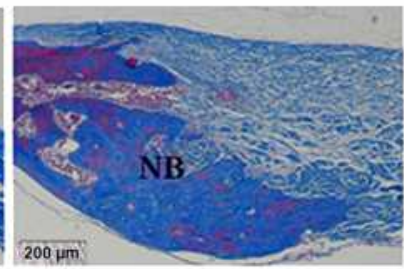

B

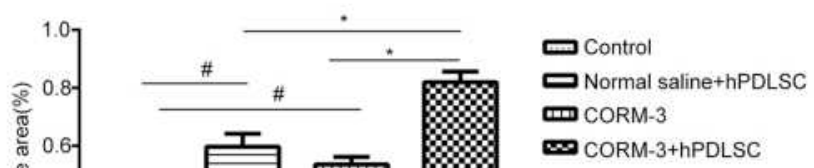

CORM-3

$\infty$ CORM-3+hPDLSC
D

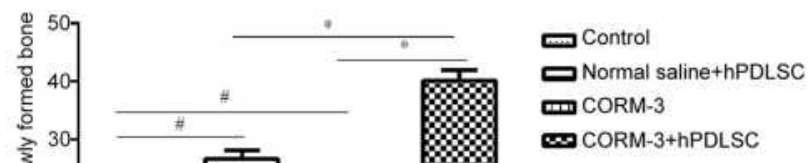

Figure 7 Effect of CORM-3 on new bone formation in nude mice with critical-sized skull defect. Animals were randomly divided into four groups. CORM-3+hPDLSC group and normal saline+hPDLSC group: defects were filled with collagen membrane seeded with hPDLSCs, in the presence (CORM-3+hPDLSC group) or absence (normal saline +hPDLSC group) of CORM-3; CORM-3 group: defects were filled with collagen membrane with the application of CORM-3; and control group: defects were filled with collagen membrane without application of CORM-3. (A and B) Four weeks after surgery, the new bone formation, and the area ratio of new bone in bone defect area was checked by H\&E staining (A). Data were quantified by Image-Pro-Plus 6.0software (B). (C and D) Masson staining was used to show new bone and the percentage of newly formed bone in bone defect region (C). Data were quantified by Image-Pro-Plus 6.0software (D). ${ }^{\#} \mathrm{P}<0.05$ vs Control; ${ }^{* P}<0.05$ as indicated.

\section{Discussion}

It has been reported that PDLSCs have the multi-lineage differentiation potential when exposed to appropriate inducing conditions, such as differentiation into cartilage, osteogenesis, and adipogenesis. ${ }^{21}$ A number of studies have revealed the favorable effects of CORMs and $\mathrm{CO}$ in in vitro and in vivo experiments. ${ }^{11}$ However, very little is known about the effect of CORMs on osteogenic differentiation of hPDLSCs. In this study, we used hPDLSCs as in vitro model to explore the effect of CORM-3 on osteogenesis of hPDLSCs. We also applied the balb/c-nude mice with critical sized skull defect as in vivo 

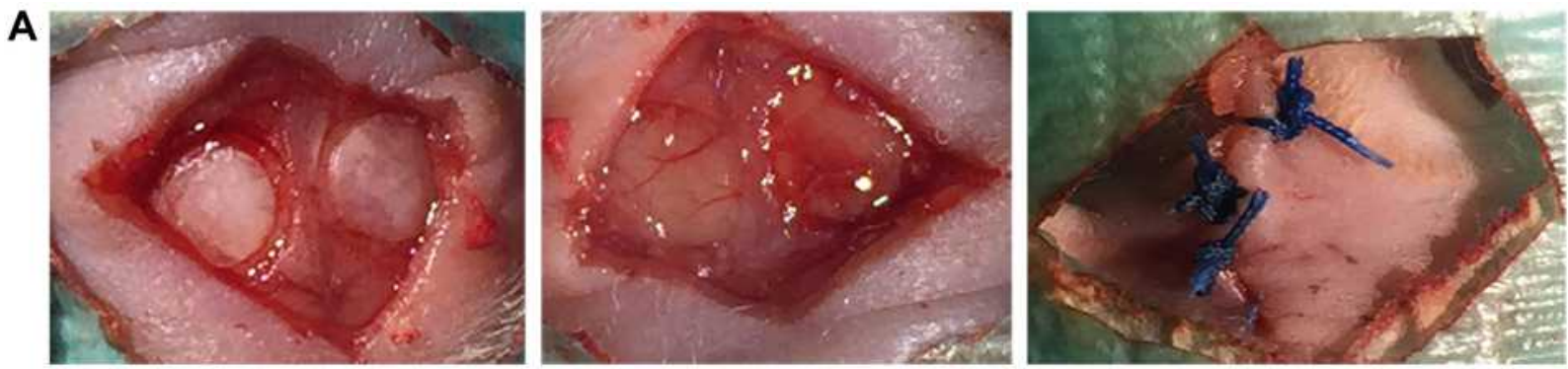

B
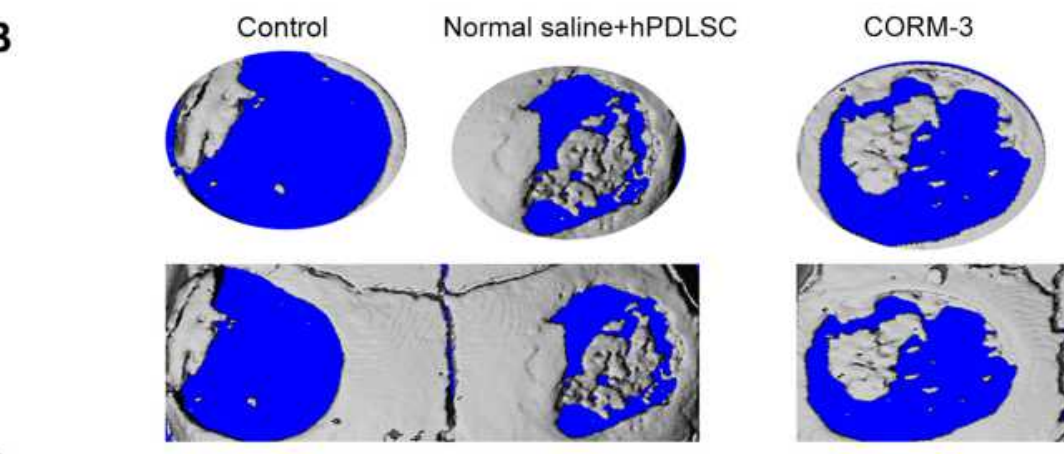

CORM-3+hPDLSC

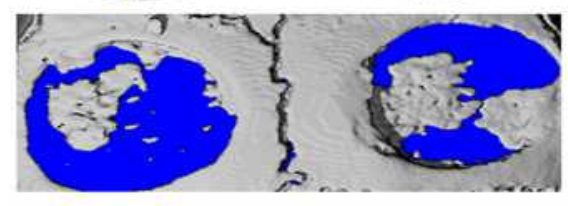

C
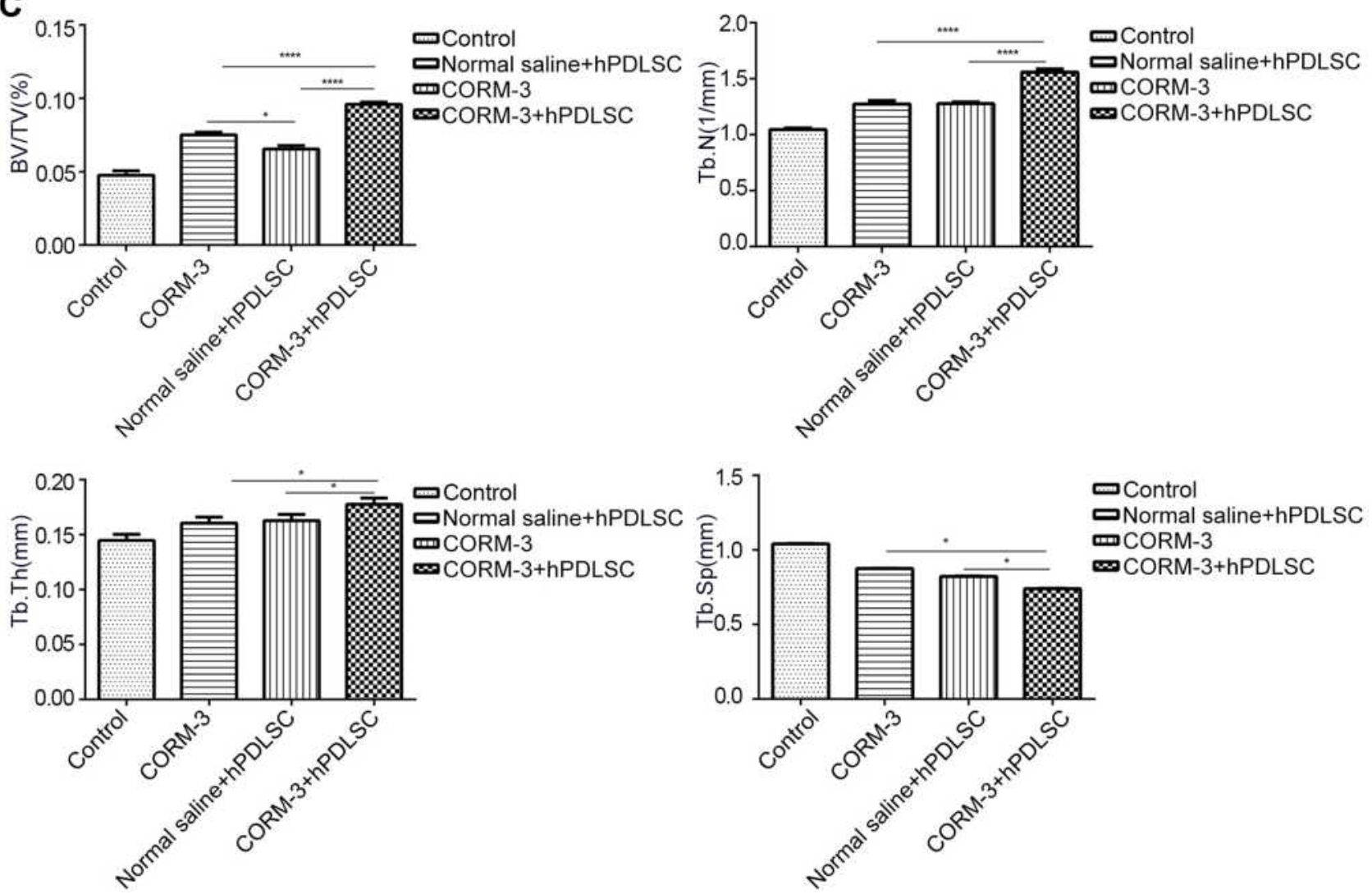

Figure 8 Effect of CORM-3 on the bone repair in nude mice with critical-sized skull defect. Animals were randomly divided into four groups. CORM-3+hPDLSC group, normal saline+hPDLSC group, CORM-3 group and control group, as described above. Bone repair in the defect area was examined by Micro-CT 7 weeks after surgery. (A) Surgical process. Skull defect with an outer diameter of $4 \mathrm{~mm}$ was established and collagen membrane (with or without hPDLSCs plantation) were transplanted into the defect region. (B) Three-dimensional micro-CT images and (C) the statistical analysis. The bone volume fraction (BV/TV, \%), trabecular number (Tb.N, I/mm), trabecular thickness (Tb.Th, mm) and trabecular separation (Tb.Sp, mm) of the new bone were measured. *P< 0.05 and $* * * * \mathrm{P}<0.000 \mathrm{I}$ as indicated. 
model to investigate whether CORM-3 together with hPDLSCs transplantation could enhance the bone repair. The results of the study were as following: firstly, CORM-3 significantly enhanced the proliferation of hPDLSCs at a concentration of $400 \mu \mathrm{M}$, which was consistent with the results of the previous study. ${ }^{22}$ Secondly, pretreatment with $400 \mu \mathrm{M}$ CORM-3 not only significantly increased the mRNA and protein expression of osteogenesis-related marker ALP, Runx2 and OPN, but also promoted the activity of ALP and the mineralization of hPDLSCs. Degassed CORM-3 did not show promotive effect on osteogenic differentiation of hPDLSCs, suggesting the modulatory effect of CORM-3 relied on the released CO. Thirdly, CORM-3 together with hPDLSCs transplantation significantly enhanced the repair of skull defects of nude mice, as evidenced by the increased new bone area and mineralization, and the augmented bone volume fraction.

Many diseases, such as infection, cancer, and degenerative diseases, etc., may cause bone resorption and defects. ${ }^{23}$ Periodontitis and periapical diseases induced inflammation may give rise to the alveolar bone defects, tooth loss, aesthetic problems, and finally affects chewing function. ${ }^{24}$ Researches have shown that PDLSCs are able to regenerate periodontal tissue, ${ }^{21}$ which suggests that the cells be an ideal source for alveolar bone repair and regeneration. ${ }^{25}$

Tissue engineering technology based on mesenchymal stem cells, scaffold materials and growth factors provide new ideas for tissue regeneration. ${ }^{26-30}$ In some bone defect diseases induced by inflammation, the inflammatory environment further increases the difficulty of bone regeneration. Searching for the novel approaches with both antiinflammation and bone regeneration enhancement capacity has therefore become a more attractive focus in the clinical treatment.

CORMs, carriers of $\mathrm{CO}$, are a kind of novel compounds which could reproduce the biological actions of $\mathrm{CO},{ }^{11}$ Studies have shown that CORMs exert anti-inflammatory effect in many cell lineages. CORM-2 has been found to inhibit the pathway of TXNIP/NLRP3 inflammasome. ${ }^{6,31}$ It was also reported that CORM-3 could suppress the expression of adhesion molecule of human gingival fibroblasts ${ }^{32}$ and inhibit osteoclastogenic and inflammatory cytokines of hPDLCs when co-stimulated by nicotine and lipopolysaccharide. ${ }^{22}$ Preclinical evidence in both large and small animal disease models confirmed the protective effects of CORMs on necrotizing enterocolitis, ${ }^{33}$ hyperacute endotoxic shock, ${ }^{34}$ and pulmonary hypertension ${ }^{35}$ at low concentration.

Based on the findings mentioned above, it was hypothesized that CORMs may not only suppress bone loss by inhibiting inflammation, but also directly promote osteogenesis and/or inhibit osteoclastogenesis. It has been widely known that Runx 2 is a critical transcription factor in early stage of osteogenesis. ${ }^{36}$ It has been found that a complete lack of bone formation on skeletal systems in fetal mice with suppression of Runx $2 .{ }^{37}$ Both OPN and ALP are osteogenicrelated markers. ${ }^{38}$ ALP plays an important role in regulating the differentiation of the cells. The increased ALP activity of the cells by CORM-3 provides beneficial conditions for the mineralization procedure which enhances the differentiation into osteoblasts. $^{39}$ The data of this study demonstrated that CORM-3 notably increased the mRNA and protein expression of ALP, OPN and Runx2, enhanced the ALP activity during the osteogenic induction of the hPDLSCs by releasing $\mathrm{CO}$, which eventually resulted in the increased osteogenic differentiation.

Previous researches have shown that the HO-1 (encoding gene HMOX1, NM_002133.3) expression is regulated in response to various stimuli, and up-regulation of $\mathrm{HO}-1$ expression enhances ALP activity. ${ }^{40,41}$ In our unpublished data, we found that CORM-3 induced high expression of HO-1, which may contribute to the increased ALP activity during osteogenesis. In clinical practice, bone distraction and transport osteogenesis have been extensively used to stimulate or enhance bone regeneration. ${ }^{42}$ However, these approaches consequently lead to healthy bone loss, deformities, infections, and chronic pains. ${ }^{43}$ The present study showed that implantation of hPDLSCs alone or CORM-3 injection alone could promote the repair of skull defect to a certain extent. The collagen membrane seeded with hPDLSCs transplantation with CORM-3 treatment showed the most prominent promotive effect on the new bone formation and mineralization. In addition, CORM-3 with hPDLSCs transplantation gave rise to a better bone microstructural system, evidenced by the increased $\mathrm{Tb}$.Th and BV/ $\mathrm{TV}$, and decreased Tb.Sp. These results provided a new idea for the treatment of bone defect diseases. Though we showed the increased new bone formation in the bone defect region by CORM-3 with hPDLSCs transplantation, we could not elucidate that the new bone was formed from the direct differentiation of the implanted cells. Fluorescence labeled hPDLSCs will be applied in our future experiment. In addition, the mechanism by which CORM-3 exerted the beneficial effect on the osteogenic differentiation and bone repair will be another focus.

Although $\mathrm{CO}$ at high concentration has been known to be toxic, many studies demonstrated that the treatment of $\mathrm{CO}$ at low concentration is feasible and effective for many diseases, 
such as chronic obstructive pulmonary disease, post-organ transplantation complications ${ }^{44}$ and hemorrhagic shock. ${ }^{45}$ Recently, hybrid molecules-termed HYCOs-by conjugating a CORM with various nuclear factor erythroid 2-related factor 2 (Nrf2) activators has been reported. CORMs which can release $\mathrm{CO}$ in target region are being explored. $\mathrm{CO}$ administration will be a promising treatment strategy in the future.

\section{Conclusion}

Taken together, our study reported that CORM-3 significantly enhanced the osteogenic differentiation of hPDLSCs by released CO. CORM-3 with hPDLSCs transplantation notably increased the repair of skull defects in nude mice. These results suggest that CORM-3 be used as an available strategy of improving bone regeneration for bone defect diseases.

\section{Abbreviations}

hPDLSCs, human periodontal ligament stem cells; ALP, alkaline phosphatase; qRT-PCR, quantitative real-time polymerase chain reaction; FBS, foetal bovine serum; PBS, phosphate buffered saline; CPC, cetylpyridinium chloride; GAPDH, glyceraldehyde-3-phosphate dehydrogenase; OPN, osteopontin; Runx2, runt related transcription factor 2; PD, Periodontitis; PMSF, phenylmethanesulfonyl fluoride; SDS-PAGE, sulfatepolyacrylamide gel electrophoresis; PVDF, polyvinylidene fluoride; SD, standard deviation; MSCs, mesenchymal stem cells.

\section{Ethics Approval and Consent to Participate}

All guidelines on animal care and use applicable to international, national and/or institutions have been complied with.

\section{Consent for Publication}

All the participants in the study obtained informed consent.

\section{Acknowledgments}

This study was supported by Shandong Provincial Natural Science Foundation (ZR2020MH186), Shandong Provincial Science and Technology Development Plan (2010GSF10270), Jinan Science and Technology Innovation Program in Clinical Medicine (201805045).

\section{Disclosure}

The authors declare no conflicts of interest in this work.

\section{References}

1. Bartold PM, Van Dyke TE. Periodontitis: a host-mediated disruption of microbial homeostasis. Unlearning learned concepts. Periodontol 2000. 2013;62(1):203-217. doi:10.1111/j.1600-0757.2012.00450.x

2. Kassebaum NJ, Bernabe E, Dahiya M, Bhandari B, Murray CJ, Marcenes W. Global burden of severe periodontitis in 1990-2010: a systematic review and meta-regression. $J$ Dent Res. 2014;93 (11):1045-1053. doi:10.1177/0022034514552491

3. Fawzy El-Sayed KM, Elahmady M, Adawi Z, et al. The periodontal stem/ progenitor cell inflammatory-regenerative cross talk: a new perspective. J Periodontal Res. 2019;54(2):81-94. doi:10.1111/jre.12616

4. Romeo L, Diomede F, Gugliandolo A, et al. Moringin induces neural differentiation in the stem cell of the human periodontal ligament. Sci Rep. 2018;8(1):9153. doi:10.1038/s41598-018-27492-0

5. Zhang X, Shan P, Otterbein LE, et al. Carbon monoxide inhibition of apoptosis during ischemia-reperfusion lung injury is dependent on the p38 mitogen-activated protein kinase pathway and involves caspase 3 . J Biol Chem. 2003;278(2):1248-1258. doi:10.1074/jbc.M208419200

6. Otterbein LE, Bach FH, Alam J, et al. Carbon monoxide has anti-inflammatory effects involving the mitogen-activated protein kinase pathway. Nat Med. 2000;6(4):422-428. doi:10.1038/74680

7. Motterlini R, Gonzales A, Foresti R, Clark JE, Green CJ, Winslow RM. Heme oxygenase-1-derived carbon monoxide contributes to the suppression of acute hypertensive responses in vivo. Circ Res. 1998;83(5):568-577. doi:10.1161/01.RES.83.5.568

8. Sammut IA, Foresti R, Clark JE, et al. Carbon monoxide is a major contributor to the regulation of vascular tone in aortas expressing high levels of haeme oxygenase-1. Br J Pharmacol. 1998;125 (7):1437-1444. doi:10.1038/sj.bjp.0702212

9. Matsumoto M, Makino Y, Tanaka T, et al. Induction of renoprotective gene expression by cobalt ameliorates ischemic injury of the kidney in rats. J Am Soc Nephrol. 2003;14(7):1825-1832. doi:10.1097/01. ASN.0000074239.22357.06

10. Sikorski EM, Hock T, Hill-Kapturczak N, Agarwal A. The story so far: molecular regulation of the heme oxygenase-1 gene in renal injury. Am J Physiol Renal Physiol. 2004;286(3):F425-F441. doi:10.1152/ajprenal.00297.2003

11. Motterlini R, Mann BE, Johnson TR, Clark JE, Foresti R, Green CJ. Bioactivity and pharmacological actions of carbon monoxide-releasing molecules. Curr Pharm Des. 2003;9 (30):2525-2539. doi:10.2174/1381612033453785

12. Foresti R, Hammad J, Clark JE, et al. Vasoactive properties of CORM-3, a novel water-soluble carbon monoxide-releasing molecule. $\mathrm{Br}$ J Pharmacol. 2004;142(3):453-460. doi:10.1038/sj.bjp.0705825

13. Wei L, Hou M, Wang P, Song H. [Effect of carbon monoxide releasing molecule on experimental periodontitis in rats]. Hua Xi Kou Qiang Yi Xue Za Zhi. 2014;32(1):23-26. Chinese.

14. Song H, Zhao H, Qu Y, et al. Carbon monoxide releasing molecule-3 inhibits concurrent tumor necrosis factor-alpha- and interleukin-1beta-induced expression of adhesion molecules on human gingival fibroblasts. J Periodontal Res. 2011;46(1):48-57. doi:10.1111/j.1600-0765.2010.01307.x

15. Li J, Song L, Hou M, Wang P, Wei L, Song H. Carbon monoxide releasing molecule3 promotes the osteogenic differentiation of rat bone marrow mesenchymal stem cells by releasing carbon monoxide. Int J Mol Med. 2018;41(4):2297-2305. doi:10.3892/ijmm.2018.3437

16. Wen Y, Lan J, Huang H, et al. Application of eGFP to label human periodontal ligament stem cells in periodontal tissue engineering. Arch Oral Biol. 2012;57(9):1241-1250. doi:10.1016/j. archoralbio.2012.02.017

17. Jiang B, Wen Y, Huang H, et al. [Study of labeling human periodontal ligament stem cells with enhanced green fluorescent protein by lentivirus vector infection]. Hua Xi Kou Qiang Yi Xue Za Zhi. 2012;30(1):82-86. Chinese. 
18. Livak KJ, Schmittgen TD. Analysis of relative gene expression data using real-time quantitative PCR and the 2(-Delta Delta $\mathrm{C}(\mathrm{T})$ ) method. Methods. 2001;25(4):402-408. doi:10.1006/meth.2001.1262

19. Lv L, Wang Y, Zhang J, Zhang T, Li S. Healing of periodontal defects and calcitonin gene related peptide expression following inferior alveolar nerve transection in rats. $J$ Mol Histol. 2014;45 (3):311-320. doi:10.1007/s10735-013-9551-2

20. Yu X, Liu H, Liu S, et al. Periodontal ligament-associated protein-1 gets involved in experimental periodontitis. J Periodontal Res. 2019;54(2):180-189. doi:10.1111/jre.12618

21. Seo BM, Miura M, Gronthos S, et al. Investigation of multipotent postnatal stem cells from human periodontal ligament. Lancet. 2004;364(9429):149-155. doi:10.1016/S0140-6736(04)16627-0

22. Song L, Li J, Yuan X, et al. Carbon monoxide-releasing molecule suppresses inflammatory and osteoclastogenic cytokines in nicotineand lipopolysaccharide-stimulated human periodontal ligament cells via the heme oxygenase-1 pathway. Int $J$ Mol Med. 2017;40 (5):1591-1601. doi:10.3892/ijmm.2017.3129

23. Loesche WJ, Grossman NS. Periodontal disease as a specific, albeit chronic, infection: diagnosis and treatment. Clin Microbiol Rev. 2001;14(4):727-752, table of contents.

24. Prince M, Banerjee C, Javed A, et al. Expression and regulation of Runx2/ Cbfa1 and osteoblast phenotypic markers during the growth and differentiation of human osteoblasts. $J$ Cell Biochem. 2001;80(3):424-440. doi:10.1002/1097-4644(20010301)80:3<424::AID-JCB160>3.0.CO;2-6

25. Aly LA. Stem cells: sources, and regenerative therapies in dental research and practice. World J Stem Cells. 2015;7(7):1047-1053. doi:10.4252/wjsc.v7.i7.1047

26. Nagata $M$, Iwasaki K, Akazawa $K$, et al. Conditioned medium from periodontal ligament stem cells enhances periodontal regeneration. Tissue Eng Part A. 2017;23(9-10):367-377. doi:10.1089/ten. tea.2016.0274

27. Zhang H, Liu S, Zhu B, Xu Q, Ding Y, Jin Y. Composite cell sheet for periodontal regeneration: crosstalk between different types of MSCs in cell sheet facilitates complex periodontal-like tissue regeneration. Stem Cell Res Ther. 2016;7(1):168. doi:10.1186/ s13287-016-0417-x

28. Ripamonti U. Redefining the induction of periodontal tissue regeneration in primates by the osteogenic proteins of the transforming growth factor-beta supergene family. J Periodontal Res. 2016;51 (6):699-715. doi:10.1111/jre.12356

29. Kononen E, Gursoy M, Gursoy UK. Periodontitis: a multifaceted disease of tooth-supporting tissues. J Clin Med. 2019;8(8):1135. doi: $10.3390 / \mathrm{jcm} 8081135$

30. Liu J, Ruan J, Weir MD, et al. Periodontal bone-ligament-cementum regeneration via Scaffolds and stem cells. Cells. 2019;8(6):537. doi: $10.3390 /$ cells 8060537

31. Jiang L, Fei D, Gong R, et al. CORM-2 inhibits TXNIP/NLRP3 inflammasome pathway in LPS-induced acute lung injury. Inflamm Res. 2016;65(11):905-915. doi:10.1007/s00011-016-0973-7

32. Zhao H, Hou M, Wei L, Mu P, Song H, Yang P. [Mechanism of carbon monoxide affecting the expression of cellular adhesion molecule under stimulation of inflammatory cytokines to human gingival fibroblasts]. Hua Xi Kou Qiang Yi Xue Za Zhi. 2013;31(4):420-424. Chinese.

Drug Design, Development and Therapy

\section{Publish your work in this journal}

Drug Design, Development and Therapy is an international, peerreviewed open-access journal that spans the spectrum of drug design and development through to clinical applications. Clinical outcomes, patient safety, and programs for the development and effective, safe, and sustained use of medicines are a feature of the journal, which has also
33. Zuckerbraun BS, Otterbein LE, Boyle P, et al. Carbon monoxide protects against the development of experimental necrotizing enterocolitis. Am J Physiol Gastrointest Liver Physiol. 2005;289(3): G607-G613. doi:10.1152/ajpgi.00055.2005

34. Mazzola S, Forni M, Albertini M, et al. Carbon monoxide pretreatment prevents respiratory derangement and ameliorates hyperacute endotoxic shock in pigs. FASEB J. 2005;19(14):2045-2047. doi:10.1096/fj.05-3782fje

35. Zuckerbraun BS, Chin BY, Wegiel B, et al. Carbon monoxide reverses established pulmonary hypertension. J Exp Med. 2006;203 (9):2109-2119. doi:10.1084/jem.20052267

36. Schroeder TM, Jensen ED, Westendorf JJ. Runx2: a master organizer of gene transcription in developing and maturing osteoblasts. Birth Defects Res C Embryo Today. 2005;75(3):213-225. doi:10.1002/bdrc.20043

37. Komori T, Yagi H, Nomura S, et al. Targeted disruption of Cbfa1 results in a complete lack of bone formation owing to maturational arrest of osteoblasts. Cell. 1997;89(5):755-764. doi:10.1016/S00928674(00)80258-5

38. Ching HS, Luddin N, Rahman IA, Ponnuraj KT. Expression of odontogenic and osteogenic markers in DPSCs and SHED: a review. Curr Stem Cell Res Ther. 2017;12(1):71-79. doi:10.2174/ 1574888X11666160815095733

39. Wennberg C, Hessle L, Lundberg P, et al. Functional characterization of osteoblasts and osteoclasts from alkaline phosphatase knockout mice. J Bone Miner Res. 2000;15(10):1879-1888. doi:10.1359/ jbmr.2000.15.10.1879

40. Vanella L, Kim DH, Asprinio D, et al. HO-1 expression increases mesenchymal stem cell-derived osteoblasts but decreases adipocyte lineage. Bone. 2010;46(1):236-243. doi:10.1016/j.bone.2009.10.012

41. Barbagallo I, Vanella A, Peterson SJ, et al. Overexpression of heme oxygenase-1 increases human osteoblast stem cell differentiation. J Bone Miner Metab. 2010;28(3):276-288. doi:10.1007/s00774009-0134-y

42. Green SA, Jackson JM, Wall DM, Marinow H, Ishkanian J. Management of segmental defects by the Ilizarov intercalary bone transport method. Clin Orthop Relat Res. 1992;280:136-142.

43. Pang P, Shimo T, Takada H, et al. Expression pattern of sonic hedgehog signaling and calcitonin gene-related peptide in the socket healing process after tooth extraction. Biochem Biophys Res Commun. 2015;467(1):21-26. doi:10.1016/j.bbrc.2015.09.139

44. Bathoorn E, Slebos DJ, Postma DS, et al. Anti-inflammatory effects of inhaled carbon monoxide in patients with COPD: a pilot study. Eur Respir J. 2007;30(6):1131-1137. doi:10.1183/09031936.00163206

45. Zhang LM, Zhang DX, Fu L, et al. Carbon monoxide-releasing molecule-3 protects against cortical pyroptosis induced by hemorrhagic shock and resuscitation via mitochondrial regulation. Free Radic Biol Med. 2019;141:299-309. doi:10.1016/j.freeradbiomed.2019.06.031

been accepted for indexing on PubMed Central. The manuscrip management system is completely online and includes a very quick and fair peer-review system, which is all easy to use. Visit http://www. dovepress.com/testimonials.php to read real quotes from published authors. 